\title{
THE MICROWAVE LANDING SYSTEM: \\ A PRECISION APPROACH FOR THE FUTURE \\ by
}

Richard P. Arnold

Manager, MLS Program Office (AND-30)

Federal Aviation Administration

800 Independence Avenue

Washington, D.C. 20591

\begin{abstract}
In response to a report to the U.S. Congress by the General Accounting Office (GAO) in May 1988, the Federal Aviation Administration (FAA) implemented a series of studies and demonstrations designed to determine the economic and operational benefits of the Microwave Landing System (MLS). Final results from this program have been reported to Congress in accordance with language contained in U.S. Senate Report 101398 on the Department of Transportation and Related Agencies Appropriations Act for fiscal year 1991.

This paper identifies and describes key findings of the MLS Demonstration Program. The results support a decision to commence with the deployment of the MLS in accordance with international implementation schedules.
\end{abstract}

\section{BACKGROUND}

The Federal Aviation Administration is charged with both the regulation of air operations and management of the U.S. National Airspace System (NAS). Rapid growth in both passenger and cargo air transportation over the past several decades has brought the need for major expansion of our air traffic management capabilities to cope with the ever increasing demand. The primary focus of those efforts, while considering all aspects of the aviation environment, falls on the terminal area operations of our major metropolitan area airports. At these facilities, where millions of passengers and tons of cargo are transitioned to and from ever expanding business centers, and to connecting flights to other parts of the world, reliable all weather precision approach and landing capability is essential. The foreseen inability of the existing Instrument Landing System (ILS) to accommodate such an expansion led the world community, through the International Civil Aviation Organization (ICAO), to identify the increased capabilities deemed necessary to meet the demands of the future.

\section{Requirements for the 21st Century}

The functional capabilities required to meet the needs of the future are those that will maximize the safe and efficient use of the terminal area airspace and the airport. ICAO identified 38 capabilities or requirements for the future system which can be synthesized into four basic operational requirements:

- Precise position information which will enable the airborne computation of precision flight paths for use in the development of advanced procedures, including computed centerline approaches, curved-approach tracks, and multiple/variable descent paths.

Wide-area coverage to facilitate an efficient transition from the en route to the terminal phases of flight. The expanded coverage volume associated with MLS as compared to ILS is shown in Exhibit 1.

- The ability to provide service at all required locations, encompassing the requirement for adequate channel capacity, ease in ground equipment siting, and signal interference immunity.

- Operation unaffected by weather, particularly during Instrument Flight Rules (IFR) conditions, when precision approach and landing systems are most critical.

Exhibit 1 - Expanded MLS Coverage Volume and Resulting Greater Final Approach Path Flexibility

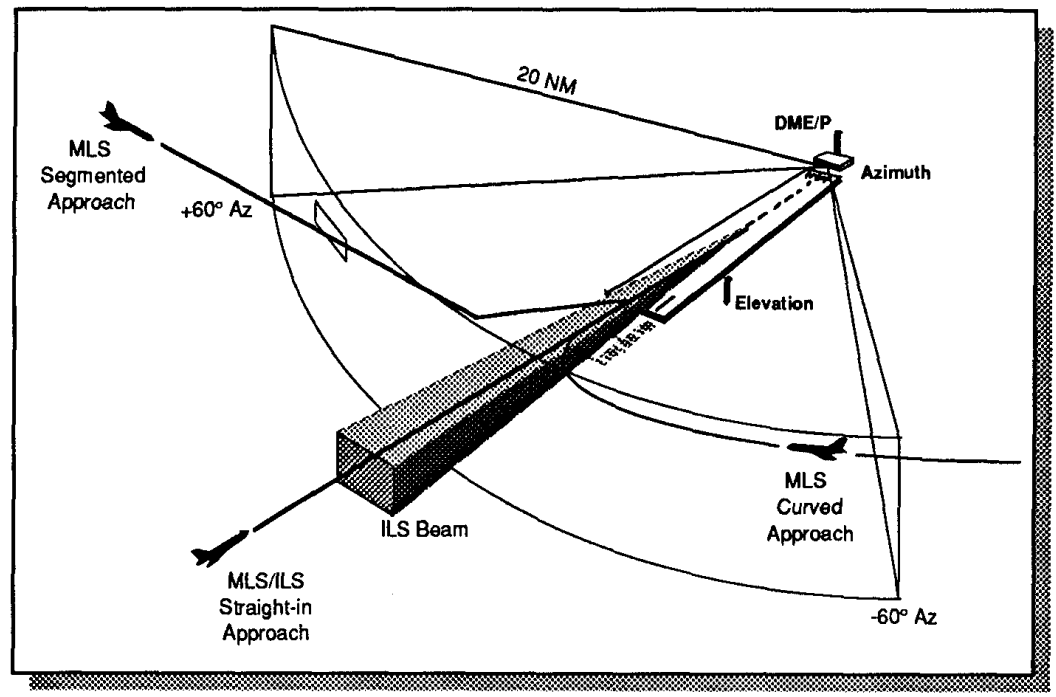


Limitations of the Instrument Landing System

The ILS is a mature technology which has served the aviation community well for nearly 50 years. The operational need for a new precision landing technology arose as a result of the inability of ILS to keep pace with future requirements. The ILS has undergone a number of improvements, improving its performance, reliability, and utility, but it does not have the fundamental design features to satisfy the operational and technical requirements of the future. Some key limitations are as follows:

- The ILS is site-sensitive. ILS uses a direct and a ground plane reflected signal to form the glideslope. Because glideslope transmitter location is directly linked to threshold crossing height and, therefore, to the touchdown area on the runway, little flexibility exists as to its placement relative to the runway and the site preparation required to accommodate it. The result is that installation costs, where extensive site preparation is required, can far exceed the cost of system hardware, especially for higher categories of service.

- Both localizer and glideslope signals are highly sensitive to nearby reflective surfaces. This necessitates more restrictive ground movement of surface traffic, including aircraft, during instrument operations which can lead to an overall reduction in airport capacity.

- Only 38 channels are available for assignment. The narrow portion of the frequency spectrum assigned to ILS severely restricts its ability to satisfy the precision approach and landing system requirements of the future. Various proposals have been made to resolve the frequency spectrum issue, to include reducing transmitter power, splitting the channels to $25 \mathrm{kHz}$ spacing, reducing the frequency protected service volume, and elimination of the VOR frequencies from the band. Each has been shown to only partially resolve the problem while at the same time adversely affecting current instrument approach procedure development standards, receiver operation and performance, and controller workload.

- ILS provides a single fixed approach path in both the lateral and vertical planes. This provides little flexibility in procedure design for purposes of deconflicting airspace, reducing noise, or avoiding terrain/obstructions. Special capability aircraft such as Short Takeoff Over Landing (STOL), rotorcraft, tiltrotor, etc., find their unique approach angle capabilities thwarted by a system incapable of taking advantage of them. Additionally, precision departure guidance cannot be provided.

Despite the extensive coordination and international acceptance of the MLS by the member nations of ICAO, problems with early U.S. procurement efforts and doubts about the costs of equipping with MLS avionics raised concerns about the entire procurement program.

\section{THE MLS DEMONSTRATION PROGRAM}

In response to the GAO report to the U.S. Congress in May 1988, the Federal Aviation Administration implemented a series of studies and demonstrations designed to determine the economic and operational benefits of the Microwave Landing System. Final results from this program have been reported to Congress. The results support a decision to commence deployment of the MLS in accordance with internationally agreed upon implementation schedules.

Both industry and Governmental agencies have participated in the planning and execution of the MLS Demonstration Program. An industry-lead MLS Task Force, that monitored the progress of the MLS Demonstration Program, requested that some projects be modified to address particular industry concerns. This included adding a detailed study of alternative precision landing technologies.

The Demonstration Program consisted of nine projects that collectively examined the operational and technological aspects of MLS. As a separate part of the evaluation, a user financial analysis was conducted to assess the benefit/cost impact that MLS operations and equipage would have on the major air carriers. What follows is a discussion of several of the key project findings.

\section{ILS/MLS Frequency Assignment Study}

The FAA contracted with the Massachusetts Institute of Technology (MIT) to perform an analysis of present and forecast ILS and MLS channel assignment capacity. The MIT developed a computer model-the Electromagnetic Simulation Applied to Landing Systems (EMSALS) - to address channel-capacity issues. EMSALS examines potential interferers and predicts their effects on airborne receivers. The product of the simulation is a listing of availability/non-availability of frequencies for new landing systems in particular locations. Frequency assignments are made in a way that optimizes use of the Radio Frequency (RF) spectrum while minimizing interference effects.

The total ILS-channel shortfall in the 10 major metropolitan areas examined ranges from 92 ( 53 percent) of the currently identified requirements to 113 (56 percent), when considering the new Denver and Chicago airports and new O'Hare runways.

An identical analysis for projected precision approach needs to the year 2010 across the continental U.S. results in a shortfall of approximately 300 ILS channel assignments, out of approximately 1,869 systems required.

A similar MLS channel-assignment evaluation resulted in a 100percent success rate, with a significant number of available channels remaining. The remaining MLS channels (as is the case with those already assigned) can be used several times in the same geographic region.

\section{Results Summary}

The MIT modeling effort shows that ILS channel-congestion and FM radio RF-interference problems will cause future channelassignment problems as follows: 
- Overall, fewer than half ( 47 percent) of the currently identified requirements for new ILS's can be satisfied in areas with high runway densities.

- The shortfall in ILS channel assignments is most severe for the New York-Philadelphia-Washington, D.C., area, where 23 out of 41 ILS channel-assignment requirements cannot be met. Attempts were made to improve the number of assignments for designated hub areas (e.g., New York). Success was limited and was achieved at the expense of reducing the number of assignments for other hub areas.

- No channel assignments are possible for the additional runways planned for Phase II operation of the new Denver airport.

- No channel assignments are available for the planned new runways at $\mathrm{O}^{\prime} \mathrm{Hare}$ Airport.

- Only half of the ILS channel-assignment requirements for a new Chicago airport can be satisfied.

- Out of approximately 1,869 precision landing systems required in 2010 , there will be a shortfall of approximately 300 ILS channel assignments.

\section{Evaluation of Wide-Body Curved Approaches}

Interest has been shown by industry regarding the flyability of curved approaches with short final straight segments, especially in wide-body aircraft. The FAA implemented two projects to address these issues. The objectives were to:

- Demonstrate the flyability of MLS curved approaches in wide-body aircraft by collecting pilot performance data.

- Evaluate pilot perception and operational acceptability of manually flown curved approach procedures.

To address these issues Boeing 747-200 and MD-11 simulations were commissioned. The B-747 simulations were performed by the Netherlands National Research Laboratory (NLR) and the MD-11 simulations were performed by the Douglas Aircraft Company.

Aircrews for the simulations were provided by industry organizations such as ATA, ALPA, and their international affiliates. Subject pilots had some knowledge of MLS based on their professional and personal readings and conversations with other industry pilots. Initial questionnaires were completed by each subject pilot to gauge crew member background, experience, and attitude regarding MLS. Subject pilots represented both international and U.S. carriers. During 1989 testing at the NLR, 16 of 20 crews were from the United States. In 1990 , all but 8 of the 20 crews represented foreign carriers. The MD-11 subject pilots were all from U.S. carriers and were similarly pre-surveyed.

NLR Simulation Description and Results Summary In 1989, four of the procedures used were to Amsterdam's
Schipol Airport, and a fifth emulated the New York JFK Canarsie approach. The four Schipol approaches were flown to final approach straight segment lengths between 3.0 and $1.5 \mathrm{NM}$ with a 90 degree left tum onto final approach. The JFK Canarsie approach used a 92 degree right tum onto final approach. In 1990 , four new approach procedures were developed to explore different variables, new topics, or address questions that arose during the 1989 test such as: precision departure capability, failures of equipment without warning during the approach, and sidestep maneuvers designed to evaluate the ability to avoid noise sensitive areas through procedure design.

Quantitative and qualitative data were collected including course tracking accuracy, pilot "feel," and a variety of subjective analyses.

During the 1989 NLR tests, 320 approaches were flown, each resulting in a landing. In 1990, 266 curved approaches and 84 departures were flown. The tests demonstrated that:

- Pilots could fly MLS-curved approaches to the same precision as current ILS approaches.

- A high level of acceptance was reflected in the pilot comments.

Pilot acceptance for a minimal straight final segment after a curved approach indicated that all pilots would accept a $3 \mathrm{NM}$ final straight segment length for revenue based flights. There was an 80 percent acceptance rate for a $2.0 \mathrm{NM}$ final straight segment and a 40 percent acceptance for a $1.5 \mathrm{NM}$ final straight segment.

Pilots rated the curved approaches as only slightly more difficult than straight-in precision approaches and as or less difficult than non-precision (i.e., no or unusable glideslope guidance information) approaches. Overall, pilot response before and after the flight trials went from "slightly negative" to "very positive" for one subject and from "unknown" to "Great!" for another. Aircrews noted that their performance improved throughout the two days of testing and their ability to anticipate the required maneuvers increased significantly with practice.

Douglas Aircraft Company MD-11 Simulations

Subjective data were evaluated such as pilot workload, flyability, and acceptability for various curved approaches to Washington National, JFK, and LaGuardia airports. The subject pilots characterized the MLS-curved approach as similar in difficulty to a non-precision approach. The only negative comments received centered on the difficulties associated with low-level precision flight with multiple turns such as the Washington National river approach. Acceptability ratings were high, ranging from $\mathbf{5 0}$ percent for the Washington National approach to 90 percent for the LaGuardia curved approach.

\section{Evaluation of Advanced Procedures in Multi-Airport Environments}

The MLS offers an opportunity to lessen or even eliminate many of the problems associated with adverse weather in a multi- 
airport environment. The primary objective of this project was to determine the operational and economic benefits of MLS in a congested terminal area environment.

The NASA Ames Research Center, Man-Vehicle Systems Research Facility conducted the simulations using air traffic controllers from the subject terminal areas and Boeing 727 pilots. The simulations were structured to model the operational environments of both the controller and the pilot who interact in the terminal area. Exhibit 2 shows the simulation in progress.

A varying mix of aircraft types, weather conditions, and landing configurations (i.e., runways in use, procedures, and aircraft spacing) were evaluated. The percentage of aircraft equipped with MLS and ILS avionics was varied for each run.

\section{New York Simulation Description and Results}

Operations at three airports in the New York Terminal Area were simulated: LaGuardia, JFK, and Newark. Under Visual Flight Rules (VFR), traffic flows to all three airports without interfering with each other. However, under IFR, with airports forced to use ILS-equipped runways, nearby Teterboro is effectively closed when LaGuardia is forced to land on Runway 13. LaGuardia approaches to Runway 13 also affect departure operations at Newark Airport. Further, an IFR approach at JFK to Runway $13 \mathrm{R}$ is not available at this time, which decreases traffic flow rates during severe weather.
Using curved approach procedures developed for LaGuardia (Runways 13 and 31), JFK (emulating the Canarsie approach to Runway 13R), and Newark (Runway 11 , currently uninstrumented), the simulation showed a clear improvement in the terminal area arrival rate. Benefits were quantified based on shorter approach path lengths and reduced airborne and ground delays.

The economic benefit from these simulations using 1990 traffic levels was predicted to be over $\$ 29$ million annually for the New York Terminal Area with 100 percent of aircraft equipped with MLS. However, with only 28 percent of aircraft MLS equipped, annual benefits were estimated to be nearly $\$ 16$ million. There was an overall reduction in flight time of more than 6 minutes per aircraft.

The Chicago and San Francisco Simulations

The Chicago simulation examined the effects of the interaction of O'Hare Airport and Midway Airport traffic during IFR conditions. It also addressed the pacing effect that $\mathrm{O}^{\prime}$ Hare Airport has on the U.S. air traffic flow as a whole.

The San Francisco simulation addressed problems associated with closely spaced parallel runways and neighboring airports (e.g., Oakland, Moffett Field). Significant reductions in delay were experienced with MLS operations even though shorter path lengths benefits were minimal. A 50 percent MLS equipage rate produced nearly the benefit that a 100 percent rate produced.

Exhibit 2 - NASA Ames Air Traffic Control Simulation Facility

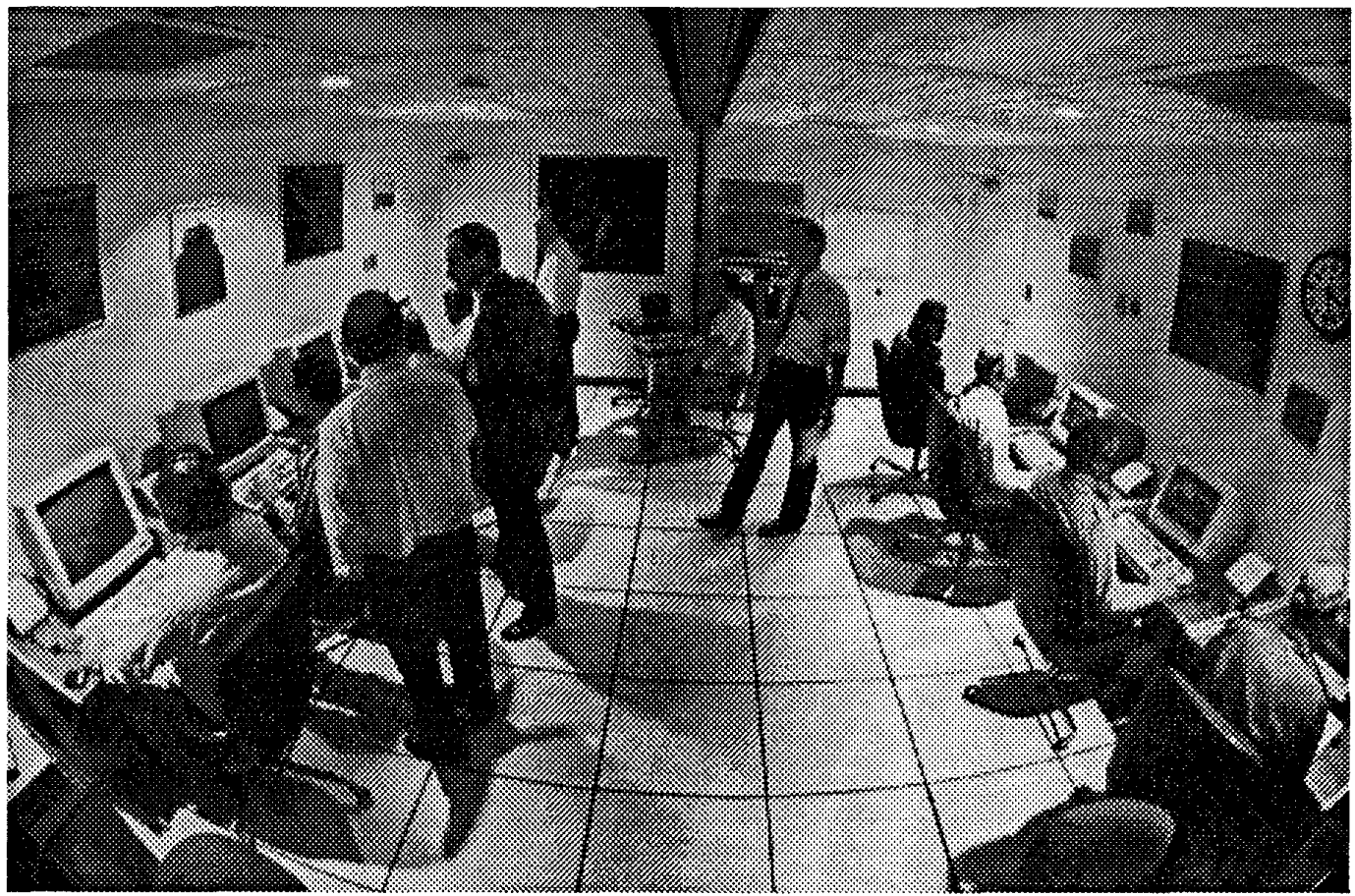


The overall benefit predicted for MLS usage in the San Francisco area was $\$ 20$ million annually using 1990 traffic levels.

\section{Assessment of MLS Alternatives}

\section{ILS and MLS Performance Comparison}

A signal-in-space performance comparison of newly improved ILS equipment versus MLS equipment was performed at several "ideal" and "trouble" sites around the country. The overall objective for these flight tests was to develop comparison data and ease of siting data for the MLS and ILS.

Eight sites were selected where the installation of an MLS ground station could operate in tandem with an existing ILS facility. These sites ranged from the "ideal" ILS site at Tamiami Airport outside Miami, Florida to a difficult, mountainous terrain site at Charleston, West Virginia. Other sites included such locations as Yuma, Arizona; Roanoke, Virginia; and JFK, New York.

\section{Comparison Results}

During the Tamiami flight tests, ILS data displayed perturbations of the landing signal when other aircraft over flew the ILS ground equipment. During these same flights, the MLS did not suffer any degradation of data. In addition the MLS data appeared to be more accurate, although the ILS did meet specifications.

Testing at Charleston, West Virginia, showed a significant difference between MLS and ILS performance. Characterized by mountainous terrain and an end-fire ILS glideslope system, the Charleston site is an ideal candidate for the versatility of a high frequency scanning beam system such as MLS. Results showed that there were ILS glideslope excursions on approach that surpassed $+/-0.50$ degrees while MLS elevation excursions rarely were greater than $+/-0.15$ degrees. A subject test pilot commented, "There was an easily discernible improvement in the flyability of the MLS over the ILS."

In accordance with ICAO documentation, recommended critical areas for ILS are greater in size than MLS. Typical critical areas for MLS are several times smaller than those for ILS. The reduced size of the MLS critical areas manifests itself in benefits such as freedom of movement for taxiing aircraft and higher ground throughput capacities at high capacity, low tarmac area airports.

\section{ILS Augmented with Area Navigation (RNAV)}

During the execution of the MLS Demonstration Programs American Airlines approached the FAA with a request to fly a curved approach during revenue operations using existing onboard systems modified for this purpose. The onboard flight management system (FMS) with multi-sensor RNAV capability would provide guidance during the curved portion of the approach.

American Airlines and the FAA undertook investigations of this subject and performed flight tests at the FAA Technical Center using a standard revenue service Boeing 757 aircraft.
RNAV Results

The protected airspace requirements for the FMS/ILS curved approach are much greater than those required for a similar MLS approach due to the non-precision input to the RNAV computer prior to ILS capture.

ILS localizer and glideslope capture of a 3.9 NM ILS final segment appeared normal to the flight crew. However, analysis of recorded data indicated glideslope capture actually occurred when the aircraft was more than 20 percent of full scale left of the localizer course. Although the localizer capture appeared routine, data analysis revealed that capture occurred, at times, more than 1.86 degrees ( 2.5 dots) from the localizer reference. At certain ILS sites this amount of angular displacement would be in a region of inverse linearity causing significant capture difficulty.

The tests were performed using an eight element log periodic array commonly used at benign sites. Based on flight test results it is unknown whether shortened final straight segments can be successfully captured at sites where 14 element localizers are used. The 14 element localizer array is designed to provide a clearance signal sector that eliminates or reduces the amount of linearly proportional localizer guidance beyond full scale deflection and is used at sites with irregular terrain (e.g. Mountainous areas) or obstructions.

Approaches to end-fire glideslope antennas (also commonly used at rough terrain sites) are characterized by reduced coverage in the horizontal plane when compared to other glideslope antenna designs. This reduced coverage in the horizontal plane manifests itself in a much narrower on-glide-path sector. It is expected that this may limit the application of FMS/LS curved approaches at end-fire glideslope sites.

Despite the limitations noted above, the tests were completed with success. Continued evaluation of the FMS/LS curved approach is essential to establishing necessary Terminal Instrument Approach Procedures (TERPS) criteria and to gain curved approach operational experience. American Airlines continues to have strong interest in FMS/ILS curved path applications and has identified additional sites where such capability would yield operational advantage. Additional performance evaluations of flight control system and FMS equipment integration will be needed before approval for use can be granted.

Additionally, an issue regarding waypoint usage (fly-by versus fly-over waypoints) must be addressed, and TERPS must be developed, describing a methodology for blending the nonprecision guidance of the FMS (or unaugmented GPS in future applications) with the precision guidance of ILS. Obstacle clearance area issues must also be addressed.

The Satellite Navigation Role In Precision Approach The aviation industry has shown enthusiasm for satellite based navigation and landing aides, focusing primarily on the United States' Global Positioning System (GPS) and the Soviet Union's Global Orbital Satellite Navigation System (GLONASS). Purportedly, with significant enhancements and modifications to 
the systems, a GPS/GLONASS system may provide precision approach capability. This topic was the subject of intensive studies sponsored by the FAA MLS Program Office.

The objectives of these investigations were to:

- Determine the feasibility of the future use of satellite navigation sources as a precision approach aid.

- Identify the technical issues to be addressed as part of a research and development program leading to a satellite based precision approach capability.

- Identify the organizational and procedural issues to be resolved for ICAO acceptance.

- Provide a projected schedule for the implementation of a GPS/GLONASS based precision approach capability.

In order to evaluate satellite capability and compare it to the MLS, three parameters were used: accuracy, integrity, and availability (continuity of service). Requirements for a satellite based precision approach were derived from existing precision approach requirements. Various permutations and augmented architectures were evaluated against these requirements.

\section{GPS Results}

Stand-alone GPS operations fail to meet every precision approach accuracy requirement by significant margins.

In order to meet CAT I precision approach requirements (the least stringent of all precision approach landing requirements) significant enhancements are required:

- Differential GPS (DGPS) with a local reference receiver and associated data link to the aircraft.

- Receiver carrier phase tracking.

- Multipath resistant antenna.

Unfortunately, as the complexity of the system increases, availability decreases. Even with enhancements, the ability to satisfy the integrity requirements remains in question.

Several proposals to enhance integrity have been proposed, to include geostationary satellites with an integrity alarm function, autonomous integrity monitoring within the receiver, and various combinations of the two. These proposals remain untested. Quantitative analysis of candidate architectures must enforce the independence of the navigation solution from the integrity function.

Availability enhancements have also been proposed including the use of geostationary satellites transmitting a GPS navigation signal, ground based psuedolites, and the use of the Soviet GLONASS satellites with GPS. Through analysis, it was quantitatively determined that all of these enhancements failed to meet availability requirements.
Work concluded thus far suggests that the GPS solution can meet Category (CAT) I accuracy requirements with enhancements. Integrity and availability continue to be problems to system designers. In addition, socio-political ramifications will come into play due to the tandem use in several candidate architectures of a combined GPS/GLONASS system.

Assuming that all technical problems and issues can be addressed and a candidate architecture could be agreed to within the next year, experts predict that the implementation of such a system cannot occur on a widespread scale until well after the turn of the century.

\section{Analysis of Synthetic/Enhanced Vision Systems}

Sensor, computer, and image processing technologies have undergone considerable technological advancements in recent years. These advancements have reinvigorated interest in the possibility of developing synthetic vision systems capable of providing visualization of terrain and obstructions to the pilot in all weather conditions. Previously, the high cost of such technology put it out of reach of the civilian user community. However, due to advances made in the synthetic vision sciences, prototype sensors for the military and civilian markets are now being developed.

The primary challenge to system developers is to produce a system that will provide a real time image of the landing area with sufficient resolution to enable a pilot to confidently land an aircraft in otherwise near zero visibility conditions. Candidate technologies include passive infrared, passive millimeter wave radiometric, and active millimeter wave systems.

The objective of this work was to assess the technical issues surrounding the introduction of synthetic vision systems, project future capabilities and applications, and develop a realistic scenario for their introduction into the NAS for civil use. Following is a partial list of unresolved issues:

- What field of view, horizontal and vertical, is required?

- How are obstacles to be treated (visually/procedurally)?

- Should the display be head up or head down and how will the pilot view be compensated for sensor versus cockpit location?

- Must the co-pilot have a synthetic vision presentation and can it be from the same source as the pilot's?

- How will integrity and continuity of service requirements be applied?

- What data is needed to augment the display for situational awareness purposes?

- What is the minimum visual range required for taxi, take off, and landing? 
In order to address these issues and others, the FAA, U.S. Department of Defense, and aviation industry representatives have undertaken extensive research into candidate architecture evaluations. Since 1989 a program has been underway to explore this technology. Currently the Synthetic Vision Program is in the analytical process. Limited results are available from the synthetic vision team. Several tasks in this phase are currently being performed including:

- Determination of technical/operational "show stoppers" which would be cause abandonment of the program.

- Learning the expansive nature and cost of the civil certification process.

- Determining how to bring the FAA, operationally and technically, into synthetic vision technology arena.

In addition the following has recently been accomplished:

- All contracts for support and equipment have been awarded.

- Four manufacturers will test equipments in 1992.

- Systems integration activity is underway and a demonstration aircraft has been selected (Gulfstream II).

\section{Future Category II/III Requirements}

There were two simultaneous efforts undertaken by the FAA MLS Program Office regarding CAT II/III MLS approaches. The first was to determine the magnitude of the requirement for future CAT II/III. The second was an effort to analytically evaluate the feasibility of establishing an intermediate CAT II minimum based upon the improved signal associated with the MLS. The improved signal quality of MLS, when used with the improved ranging accuracy of Precision DME (DME/P), offers greater flexibility in the precision approach environment over earlier systems (e.g., ILS and VOR/DME).

Analysis and evaluation of CAT II/III requirements were centered about the following tasks:

- Projected needs for CAT II/III approaches in CONUS.

- Increases in airport landing capacity under IFR.

- Which technologies (ILS, MLS or both) can satisfy needs.

- Variable costs associated with each system.

Efforts to establish a new CAT II Decision Height (DH) revolved around determining what additional systems will be required to establish a 150-foot DH over and above the equipment necessary to perform a CAT I approach (200-foot DH) today.

The overall objectives of these efforts were to:

- Determine the increased demand for additional CAT I/II runways in the CONUS.
- Validate the feasibility of achieving an intermediate 150foot CAT II DH and define the systems (i.e., ground equipment, avionics, training, and landing area environment) necessary to support it.

Results

To date over 500 runway ends have been identified for CAT II/ III landing systems by the year 2010 .

Efforts to determine the feasibility of the 150 -foot DH have resulted in the following preliminary indications:

- The stability of the MLS signal improves performance and situational awareness for the pilot.

- Pilots will accept the 150 -foot DH for revenue service operations.

- A flight director is required.

- The medium intensity approach lighting system is sufficient for these operations with runway alignment indicator lights. Without runway alignment indication, high intensity lights are needed for minimums less than 200 feet.

- Touchdown zone and center line lighting is desirable but not required.

- Radar altimeter or MLS computed height is required for determining the $\mathrm{DH}$.

\section{MLS Benefit/Cost Analysis}

There were two studies performed during the FAA MLS Demonstration Program that addressed cost and benefits associated with MLS. The first was an evaluation of projected costs of MLS equipments for aircraft and runways. Comparisons were made to current costs of ILS equipment. In the second study projected benefits at selected airports were evaluated with the addition of MLS ground equipment.

The Cost to Implement MLS vs. ILS

The overall objective of this project was to determine MLS costs and compare them with current ILS costs. Since MLS will have a significant fiscal impact on the aviation community, studies of equipment costs were undertaken.

With the participation of industry and Government experts, analyses were performed to assess MLS costs. A representative number of avionics configurations were studied and costs were determined for procurement, installation, certification, and maintenance, over a 20-year life cycle. Ground equipment costs were derived from manufacturer and current procurement estimates.

\section{Results of Cost Study}

Costs were determined in 1990 dollars (except for certification costs which are in year 2000 dollars). MLS avionics and installation costs were shown to be comparable to existing ILS avionics costs when produced in large quantities for the 
commercial market. DME/P is projected to cost $\$ 5,000$ more per unit than a DME/N.

Certification costs for the entire commercial U.S. air carrier fleet is estimated between $\$ 18$ and $\$ 26$ million. An additional $\$ 5$ to $\$ 16.3$ million will be required for regional air carrier certification. Projected general aviation certification cost is between $\$ 1,500$ and $\$ 5,000$ per aircraft.

Due to enhancements in technology, maintenance costs of MLS are expected to be equal to or less than ILS even though the complexity of MLS equipment is greater.

\section{MLS Benefits Study for Selected Airports}

An FAA study group examined current Visual Flight Rules (VFR) and IFR operations (with ILS) at four U.S. airports to determine if MLS could improve traffic flow when operating under IFR. The objective of this effort was to assign operational and economic benefits to the improved traffic flow during IFR operations with the introduction of MLS. The airports evaluated were: JFK, Chicago Midway, Philadelphia, and San Francisco International.

\section{Results}

The FAA study group determined that the 4 airports could each support approximately 52 landings per hour during VFR operations. When IFR are imposed, with existing ILS equipment, these numbers dropped to as low as 28 landings per hour. With the introduction of MLS at these airports 3 of the 4 sites returned to near 52 landing operations per hour.

\section{CONCLUSIONS}

Evaluations, projects, and analyses that determine the technical, operational, and economic benefits associated with MLS will be fully reported upon completion. Results available to date strongly favor moving forward with MLS implementation.

Increased landing rates during IFR operations, significant benefit over cost, improved safety, and expansion of the precision approach capability within the U.S. all hinge on the implementation of MLS. As stated earlier, results to date indicate:
- It will not be possible to install ILS at all the sites where a precision approach capability is needed. Significant NAS requirements for a growing aviation industry will not be met without introduction of a new precision approach capability.

- New alternatives to MLS are not mature enough to be implemented within the reasonable future. GPS, with augmentation, cannot satisfy CAT II/II requirements.

- MLS procedures can reduce weather induced delays in the New York Terminal Area resulting in projected user benefits of $\$ 30$ million annually.

- MLS avionics and ground equipment costs are comparable to existing ILS equipments.

- FMS/RNAV in the terminal environment with ILS has limited applications for curved approaches at some locations.

The MLS offers significant benefits which cannot be realized through continued application of ILS. By endorsing the ICAO ILS/MLS Transition Plan, the U.S. has committed to install MLS ground systems at those runways used by international carriers by the year 1998. Further, the U.S. Department of Defense, the single largest user of precision landing systems in the world, has committed to equip over 18,000 aircraft and several hundred runways worldwide with MLS.

The MLS design was standardized to satisfy each of the requirements identified by ICAO as key to effective precision landing system operations in the 21st century. To meet the 1998 commitment to ICAO, the FAA MLS Transition Plan requires that early production MLS systems be installed first at international runways. Subsequent systems will be installed at existing ILS-equipped runways, followed by new sites which are candidates for precision landing systems as defined in Airport Planning Standard Number One (APS-1), FAA Order 7031.2C. Included in the last category are a number of locations that do not currently have precision approach service due to ILS siting constraints. 